\title{
Pediatric Difficult Intubation: A Newborn baby with a Giant Occipital Meningoceole
}

\author{
Mozibul Haque, Abul Kalam Azad, Md Rabiul Alam \\ Combined Military Hospital, Dhaka Cantonment, Dhaka 1206, Bangladesh.
}

\begin{abstract}
Corresponding Author:
Dr (Brig Gen) Mozibul Haque

Email: colmhaque@yahoo.com
\end{abstract}

This is an Open Access article distributed under the terms of the Creative Commons Attribution License (creativecommons.org/ licenses/by/3.0).

Received Accepted Published

June 2, 2019

November 9, 2019

November 20, 2019

\begin{abstract}
Background: Cranial meningocele represents herniation of the cranial meninges containing cerebrospinal fluid through a congenital neural defect in the cranium. A meningocele poses many challenges to the anaesthesiologists when presents for surgical excision and repair. Difficult or failed intubation frequently happen due to improper positioning and limited head excursion. Case Report: We encountered a giant bilobulated occipital meningocele in an 8-day male neonate weighing 3.8 kilogram. We placed the baby supine on a firm pillow. The meningocele sac was beyond the edge of the pillow supported by appropriately sized padded "doughnut". We encountered difficult intubation as anticipated due to restricted head and neck movement.. The laryngoscopic view of the glottis initially was Cormack-Lehane Grade 3. Once the assistant pressed over the larynx the Cormack-Lehane Grade became 2 and the tracheal intubation was possible. Then the baby was placed in lateral position carefully as surgery was planned to be performed in this position. Conclusion: Anaesthetic management of a case of a giant occipital meningocele demands identification of the problems and a management plan accordingly. Anaesthesiologist sometimes may need to adopt unconventional and innovative ideas to handle such cases.
\end{abstract}

Keywords: Anesthesiologists, Intratracheal Intubation, Meningocele, Neck, Newborn.

\section{Introduction}

Airway management in newborns with craniofacial disorders always offers greatest challenges to the anaesthesiologist [1]. Many inherent factors like disproportionately sized head and body and unstable head and neck create difficulties of tracheal intubation to the anaesthesiologist. It becomes more difficult if this condition is compounded by a huge occipital mass which is almost double the size of the head. In such cases difficult or failed tracheal intubation is feared by almost all the anaesthesiologist due to improper positioning and limited neck excursion [2]. The purpose of reporting this case of a difficult intubation is to describe the problems we encountered and to share how the problems were addressed in a case of bilobulated giant occipital meningocele.

\section{Case Report}

An 8-day old male neonate weighing 3.8 kilogram presented with a giant bilobulated occipital meningocele for surgical excision and repair. The bigger lobe of the presented meningocele measured $18 \times 14 \mathrm{~cm}$ and the smaller one was about twothird in shape and size of bigger lobe [Fig.1]. The cystic swelling was soft and translucent containing fluid (CSF) and was highly mobile. It was nonreducible and attached to the occiput with a short small and narrow peduncle (stalk). There was no neurological deficit and no other discernable congenital abnormalities. In the operating room the baby was placed supine on a firm pillow and the meningocele was allowed to hang beyond the edge of the pillow. The height of the pillow was adjusted in such a manner that the meningocele 
sac just touched the mattress of the table. We then placed a properly sized padded "doughnut" under the meningeal sac to immobilize it and to avoid direct pressure on it as well. Intravenous atropine $0.02 \mathrm{mg} / \mathrm{kg}$ was given to combat reflex bradycardia to happen.

Fentanyl $2 \mu \mathrm{gm} / \mathrm{kg}$ was administered intravenously just before induction. Since we did not have the facilities of either isoflurane or sevoflurane we induced the baby with halothane starting from $0.5 \%$ upto $2 \%$ in $100 \% \mathrm{O}_{2}$ using a size 1 facemask and Jackson-Ree's medication of Ayre's T-piece. When the patient developed apnea, controlled ventilation was started and after two minutes tracheal intubation maneuver was undertaken. A small sized Miller (straight) laryngoscope blade (size 0,1 ) was introduced into the mouth at the right commissure displacing the tongue completely to the left. But the glottis did not come into view. Only the tip of the epiglottis was visible (Cormack-Lehane Grade 3). Then tip of the blade was inserted beneath the epiglottis and lifted up applying constant force along the axis of the laryngoscope handle via elbow. A second assistant then pressed over the larynx to bring the oral and laryngeal axis straight. Once the visualization of the glottis in this position improved (CormackLehane Grade 2) a $3 \mathrm{~mm}$ non-cuffed armoured ETT with stellate was introduced orally. After securing the ETT the baby was placed carefully in the lateral position on the operating table over a warm blanket as surgery was planned to be performed in lateral position. The duration of surgery was 1 hour 40 minute and was uneventful. When the baby was fully awake after discontinuation of anaesthetic gases the trachea was extubated on the operating table. The neurological integrity of the baby was checked and sent to intensive care unit (ICU) for post-operative management.

\section{Discussion}

There are many reports of difficult tracheal intubation in patients with meningocele. In

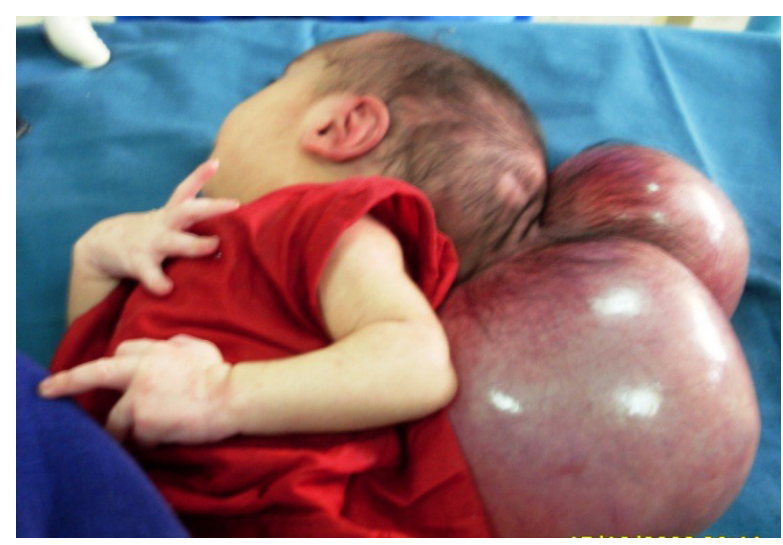

Fig.1: Bilobulated occipital meningocele.

occipital meningocele (encephalocele) the anterior and middle fossae are usually deformed with frequent association of Chiari malformation. Coexisting hydrocephalus may occur due to torsion of the aqueduct or aqueductal stenosis. Most of the cases clinically present as a midline cystic mass of variable size and shape. Many congenital conditions like facial deformities, craniofacial dysmorphologies, cystic hygroma, Treacher-Collin syndrome (Mandibulofacial dysostosis), Pierre-Robin syndrome (micrognathia with mandibular hypoplasia) are frequently associated with reduced mouth opening and neck morbidity. Airway management of small children with a giant occipital meningocele is a major anaesthetic challenge. The occipital meningocele puts restriction to the movement of the head and neck. This led to difficulty in visualization of glottis on laryngoscopy. We induced anaesthesia and tracheal intubation in supine position placing the baby on a firm pillow. The meningocele sac was beyond the edge of the pillow supported by an appropriately sized padded "doughnut". Even then we encountered difficult intubation due to restricted head and neck movement. Difficult airway management plan has been defined by various authors for occipital meningocele. Mowafi et al. described a platform method to deal with a huge posteriorly located encephalocele in supine position $[4,5]$. Our method was similar to that of described by Mowafi. We used an appropriately 
sized firm pillow instead of folded blanket under the back to prevent pressure on the meningocele sac and possible rupture. Alternative approach is to lift the baby off the table with the help of two assistant [6]. This method is not much helpful because it needs two or more assistant and difficult to stabilize the free head and neck manually.

Tracheal intubation may also be undertaken in lateral position to avoid direct pressure on the sac and to get greater mobility of the head and neck [7]. But at times it becomes more difficult to intubate a small baby in lateral decubitus position than supine [8]. Meticulous attention must be given to the position of the patient during surgery. Surgery on occipital meningocoele is sometimes performed in prone position. Despite the difficulties in airway management in prone position it confers an additional disadvantage during surgery. Any external pressure on the anterior abdominal wall is transmitted to the inferior vena cava and impedes the venous return from lower part of the body. Transverse bolsters under the chest and pelvic girdle ensure that the abdomen remains free, permitting unimpeded respiratory excursion and good venous return. Prone position for long time may result edema of the airway leading to airway obstruction after tracheal extubation. The decision to extubate the trachea in such a difficult case depends on the length of the procedure, intra-operative course of events and the extent of surgical resection.

\section{Conclusion}

Management strategy in a case of difficult tracheal intubation includes identifying the nature of difficulty and developing a management plan for the problem. Careful consideration and expert handling of the related problem are essential part of the anaesthetic management involving such cases. Anaesthesiologists in those cases may need to adopt unconventional but noble and safe ideas for safe and better management with successful outcome.

Contributors: MH: Manuscript writing, patient management. AKA: literature search, manuscript preparation, and patient management; MRA: manuscript editing, and patient management. $\mathrm{MH}$ will act as a study guarantor. All authors approved the final version of this manuscript.

Funding: None; Competing interests: None stated.

\section{References}

1. Amata OA. Posterior encephalocele: a case of difficult tracheal intubation. West Afr J Med. 1994;13(3):187190.

2. Yentis SM. Predicting difficult intubation- Worthwhile exercise or pointless ritual? Anaesthesia. 2002;57:105109.

3. Hassani MD. Anaesthesia complications in neonates with neural tube defect. European Journal of Anaesthesiology. 2008;25:1-36.

4. Mowafi HA, Sheikh BY, Al-Ghamdi AA. Positioning for anesthetic induction of neonates with encephalocele. The Internet Journal of Anesthesiology. 2001;5(3).

5. Mahan Y, Chinnan NK, Singh AK. Neonatal airway management in occipital meningocele. Anesth Analg. 2006;103:1632.

6. Singh KV, Garasia MB, Ambardekar M, Thota R, Dewoolkar. Giant occipital meningoencephalocele: Anaesthetic implications. The Internet Journal of Anesthesiology. 2007;13:2.

7. Geol V, Dogra N, Khandelwal M, Chaudhri RS. Management of neonatal giant occipital encephalocele: Anaesthetic challenge. Indian J Anaesth. 2010;54(5):477478. 